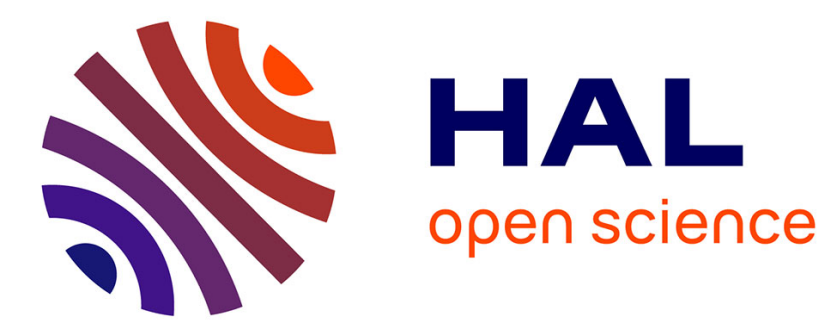

\title{
Production écrite autour de questions éthiques en langue de spécialité
}

\author{
Joséphine Rémon
}

\section{To cite this version:}

Joséphine Rémon. Production écrite autour de questions éthiques en langue de spécialité. Recherche et Pratiques Pédagogiques en Langues de Spécialité: Cahiers de l'APLIUT, 2013, 32 (2), pp.124-140. 10.4000/apliut.3805 . hal-00866980

\section{HAL Id: hal-00866980 https://hal.science/hal-00866980}

Submitted on 27 Sep 2013

HAL is a multi-disciplinary open access archive for the deposit and dissemination of scientific research documents, whether they are published or not. The documents may come from teaching and research institutions in France or abroad, or from public or private research centers.
L'archive ouverte pluridisciplinaire HAL, est destinée au dépôt et à la diffusion de documents scientifiques de niveau recherche, publiés ou non, émanant des établissements d'enseignement et de recherche français ou étrangers, des laboratoires publics ou privés. 


\section{Production écrite autour de questions éthiques en langue de spécialité}

Mots clés :

prise de risque langagière, questions vives, langue de spécialité, didactique de l'anglais, motivation, éthique

\section{Résumé :}

Dans le cadre d'une interrogation sur la place de l'éthique dans l'apprentissage d'une langue de spécialité, nous nous penchons sur l'utilisation de questions éthiques en pratique de la langue, à la lumière de la notion de prise de risque langagière. Nous faisons l'hypothèse que l'utilisation de questions éthiquement vives lors d'activités de production langagière peut contribuer à cette prise de risque, elle-même synonyme d'engagement dans la tâche. Nous menons une étude de corpus qualitative de productions écrites provenant de séances avec des étudiants en Master Communication humanitaire et en Master Sciences de l'éducation. La prise de risque langagière apparaît à travers des marques énonciatives d'engagement dans la tâche telles que l'humour ou la modalité et à travers la complexité du propos des étudiants, malgré leurs moyens linguistiques limités.

\section{Written production around ethical questions in language for specific purposes}

Key words:

language risk-taking, acute questions, language for specific purposes, English teaching, motivation, ethics

\footnotetext{
Abstract:

In the wider context of the study of the role of ethics in language learning for specific purposes, we focus on the use of ethical questions in language practice, from the point of view of language risk-taking. We follow the hypothesis that using ethically acute questions for language production activities can contribute to this risk-taking, itself a sign of engagement in the task. We undertake a qualitative study of a corpus of written productions from classes with students working towards a Master either in Humanitarian communication or in Educational sciences. Language risk-taking appears through enunciative traces of engagement in the task, such as humour and modality, and through complex reasoning on behalf of the students in spite of their limited linguistic means.
} 


\title{
Production écrite autour de questions éthiques en langue de spécialité
}

\begin{abstract}
À travers une étude qualitative de productions écrites d'étudiants, nous nous proposons d'aborder les questions d'éthique dans l'enseignement des langues de spécialité sous l'angle de la prise de risque langagière. Nous étudions comment, même avec des moyens langagiers limités, les étudiants font preuve d'un fort engagement dans la tâche et parviennent à s'exprimer sur des sujets complexes. Nous relevons dans le corpus des traces relevant du fond, du raisonnement mis en œuvre par les apprenants, et d'autres traces relevant de la forme, telles que la modalité, l'humour et la phraséologie. La transversalité des questions éthiques permet de les utiliser non plus comme prétexte à la pratique langagière mais d'utiliser la classe de langue pour penser l'éthique, l'engagement dans la tâche didactique se prolongeant dans un engagement citoyen.
\end{abstract}

\section{1 Éthique et enseignement des langues}

Nous nous penchons ici non pas sur une éthique professionnelle pour des étudiants en tant que futurs acteurs du monde du travail, mais sur une éthique citoyenne transversale invoquée dans le cadre de débats dans la classe de langue de spécialité. L'éthique telle qu'elle est envisagée ici n'est pas l'apprentissage des bonnes mœurs (Forestal 2009 : 111) ou d'une morale individuelle, mais l'apprentissage de règles de conduite de l'apprenant, sujet-pensant citoyen, autour d'une visée éthique, «visée de la 'vie bonne' avec et pour autrui dans des institutions justes $»^{1}$ (Forestal 2009 : 72). Les sujets de réflexion proposés aux étudiants interrogent le rapport de chacun à soi-même et aux autres. Dès lors la notion de respect est centrale : « respect (par l'enseignant) de l'autre (l'élève) et respect (par l'élève et l'enseignant) de l'engagement pris (par l'élève et l'enseignant)» (Puren $1994: 6$ ). L'interrogation de ces règles éthiques est inhérente à la didactique des langues en général, qui « est en droit et a le devoir de se poser la question essentielle de notre survie et de notre avenir en tant qu'espèce humaine » et doit «faire de la morale sans faire la morale » (Forestal 2009 : 71).

Nous rapprochons ces questions éthiques des questions dites «socialement vives » (Legardez \& Simmoneaux 2006). Débats et controverses font partie de l'éducation scientifique et citoyenne (Simonneaux \& Legardez 2010:31), l'éducation à la citoyenneté comprenant des aspects cognitifs, sociaux et éthiques (Audigier 1999). L'utilisation de questions éthiques participe d'une pédagogie de l'étonnement (Mathieu 2010), de la provocation ou du paradoxe. Cette utilisation introduit l'« imprudence disciplinaire » de Forestal (2009: 71) qui fait de la classe de langue « le lieu d'une pratique à risques. La gestion du conflit par la parole peut, on le sait, avoir des effets positifs mais aussi des effets négatifs »(Forestal 2007 : 122). Cette

\footnotetext{
${ }^{1}$ Ricoeur P. (1990)Soi-même comme un autre (1990). 1990 :202
} 
pédagogie du conflit privilégie «la polémique, le débat, l'argumentation » et développe «l'esprit critique, l'honnêteté intellectuelle, le courage de communiquer ses sentiments, d'aller au-delà de soi, de coopérer, de partager les tâches, mais aussi de reconnaître ses limites ou celles du groupe et d'accepter des situations dans lesquelles aucune solution constructive ne peut être trouvée» (idem: 119). L'utilisation de «questions éthiquement vives» (Rémon 2012) nous semble permettre la «mise en scène renouvelée du savoir » (Astolfi 2006 : 9) nécessaire à la motivation. Cette mise en scène offre la diversion qui permet, au même titre que l'humour par exemple, de focaliser sur autre chose que la langue pour elle-même (Scrivener 2011 : 328).

Nos hypothèses se déclinent donc de la manière suivante :

- la prise de risque langagière (Rémon 2010) contribue à l'efficacité de l'apprentissage ;

- la prise de risque langagière est favorisée par des facteurs réduisant l'anxiété linguistique ;

- des «diversions » permettant de focaliser sur un autre objet que la langue en elle-même favorisent la réduction de l'anxiété linguistique ;

- la réduction de l'anxiété linguistique favorise l'engagement dans la tâche ;

- la réflexion éthique constitue une diversion permettant de focaliser sur autre chose que la langue en elle-même et contribue à l'engagement dans la tâche et à la prise de risque langagière.

Par prise de risque langagière, nous entendons l'utilisation de la langue cible en dehors de la zone de confort de l'apprenant, à travers des structures dont l'apprentissage n'est pas encore stabilisé, et des éléments complexes tels que l'expression de l'humour ou la phraséologie. Les marques de cette prise de risque langagière sont présentes typiquement, selon nous, lorsqu'il y a tentative d'utiliser une expression phraséologique en faisant une erreur. Ces éléments sont relevés dans un contexte où l'objectif pédagogique est la pratique de la langue et la prise de confiance. Nous ne sous-entendons pas que ce contexte permet l'apprentissage à travers des échanges de propos erronés mais que cette pratique de la langue est l'un des aspects d'un dispositif pédagogique qui comprendra par ailleurs un retour sur la structure de la langue.

\section{Présentation du corpus}

Notre corpus est composé de productions écrites autour de questions éthiques dans deux contextes différents avec trente étudiants dans les deux cas : 8 débats en ligne d'environ 50 contributions chacun entre étudiants en Master Sciences de l'éducation dans le cadre d'une formation à distance dont le module TICE-anglais dure huit semaines, et une séance de production écrite d'une demi-feuille en anglais en présentiel avec des étudiants en Master Communication humanitaire et solidarité dans le cadre d'un module d'anglais de 30 heures.

\subsection{Débat synchrone en ligne}


Dans le cadre du module TICE-anglais d'un Master à distance en Sciences de l'éducation, les étudiants doivent accomplir diverses tâches impliquant la pratique de la langue et la pratique de la technologie autour d'une plateforme d'apprentissage. Dans le cadre d'une de ces tâches, ces étudiants de Master 1 se retrouvent en ligne, par petits groupes en fonction de leurs disponibilités horaires, pour tester un outil d'apprentissage de débat (http://scale.emse.fr/pws/). Ils produisent ensuite un compterendu écrit qu'ils publient sur la plateforme d'apprentissage. L'outil de débat se compose d'une fenêtre où les étudiants argumentent sous la forme de boîtes et de flèches et d'une fenêtre de clavardage destinée aux commentaires (Figure 1).

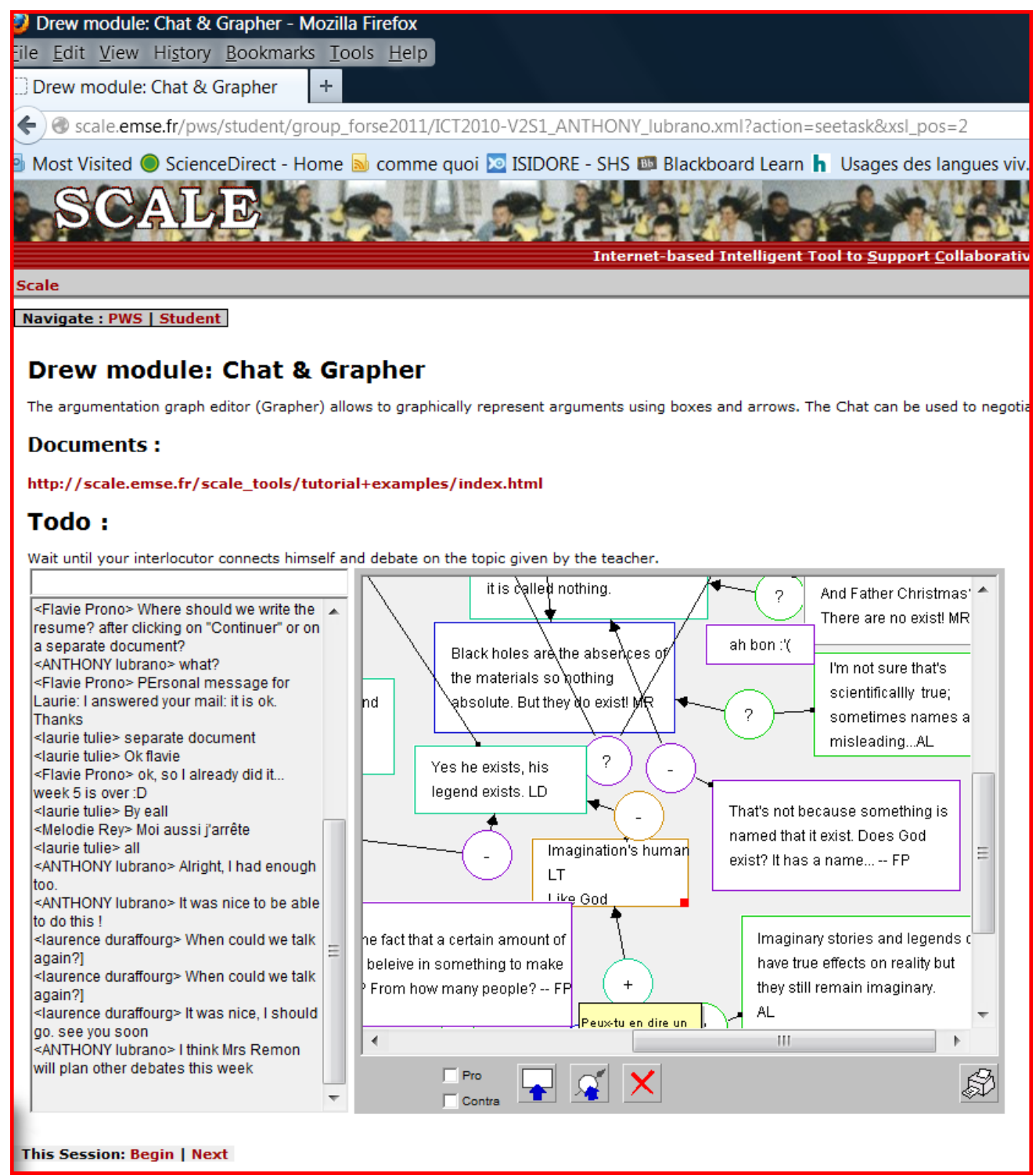


Figure 1 : Saisie d'écran montrant la fenêtre de clavardage et la fenêtre de construction du débat.

Le corpus qui en résulte est donc composé des graphes créés par les étudiants et des échanges dans la fenêtre de clavardage. La construction des graphes peut être rejouée au ralenti et des fichiers générés automatiquement décomposent aussi les graphes sous forme textuelle.

Les thèmes utilisés pour le débat sont issus d'un ouvrage appelé Little book of Thunks par Ian Gilbert (2007). Ce sont, par exemple :

- Si un somnambule tue pendant son sommeil, est-il responsable?

- Est-ce que vous échangeriez votre sens de l'humour contre 1 million de dollars?

- Est-ce que les idiots doivent être autorisées à voter ?

- Peut-on justifier de harceler un harceleur ?

- Si vous étiez identifié comme étant génétiquement programmé pour faire de mauvaises actions, faudrait-il vous enfermer avant que cela arrive ?

- Si vous pouviez presser un bouton pour faire mal à quelqu'un sans que quiconque le sache, le feriez-vous pour 1 million de dollars ?

Une ou deux des propositions utilisées ont un caractère plus généralement philosophique et sont moins provocatrices du point de vue éthique comme «Est-il possible de ne rien faire ? » ou «Est-ce qu'un nouveau-né aime sa mère ? ». Un exemple avec une thématique moins directement liée à l'éthique montre que la motivation est absente, et que l'on revient à la pratique de la langue pour elle-même : à propos du sujet de débat «Est-ce qu'une feuille de papier blanche est plus vide qu'une feuille noire ?» nous avons recueilli cet échange :

$$
\begin{aligned}
& \text { 1. } \quad<\mathrm{jm}>\mathrm{i} \text { admitt that this topic does not inspire me! Sorry }{ }^{2} \\
& <\mathrm{cv}>\mathrm{it} \text { 's the same for me...but it's just for the exercice... } \\
& <\mathrm{jm}>\text { yes of course! }
\end{aligned}
$$

\subsection{Productions écrites sur papier}

Le deuxième corpus est constitué de productions écrites provenant de séances d'anglais avec des étudiants en Master Communication humanitaire et solidarité. Lors d'une de ces séances, nous les amenons d'abord à s'interroger sur les principes éthiques régissant le secteur humanitaire, puis ils exploitent des documents écrits et audio en anglais relatifs à l'expérience de psychologie sociale d'obéissance à l'autorité de Milgram qui mêle éthique et psychologie (les participants infligent des chocs électriques potentiellement mortels à un «élève » à la demande d'une figure d'autorité). Enfin, on leur propose en anglais un sujet de réflexion, tiré de l'ouvrage de Ian Gilbert (2007) : «Si vous pouviez presser un bouton pour faire mal à quelqu'un sans que quiconque le sache, le feriez-vous pour 1 million de dollars ?». Ils écrivent quelques

\footnotetext{
${ }^{2}$ Les extraits introduits par des initiales entre crochets $<>$ sont issus de la fenêtre de clavardage dans le débat à distance, tandis que les extraits sans crochets sont issus du débat en ligne ou de la séance de production écrite en présentiel. Les extraits sont traduits en Annexe 1.
} 
phrases à partir de cette question, sans aucun enjeu d'évaluation. Avec ce corpus, les facteurs d'engagement qui pourraient être liés à la distance et à l'utilisation d'un logiciel de débat en ligne sont neutralisés, puisqu'il s'agit d'un exercice en présentiel sur papier.

\section{Engagement dans la tâche et prise de risque langagière}

Quel que soit le corpus, les mêmes caractéristiques (telles que l'humour ou la modalité) apparaissent et se divisent entre des signes de l'énonciateur dans son discours et des éléments qui portent sur le fond et montrent la complexité du propos malgré des moyens linguistiques parfois faibles. La prise de risque apparaît donc à travers les erreurs linguistiques, en dépit d'obstacles langagiers, et en dépit d'obstacles matériels dans le cas de la formation à distance.

\subsection{Difficultés linguistiques et logistiques}

En effet, les signes d'engagement que l'on peut repérer ont d'autant plus de signification que l'activité, pour certains, n'est pas facile. Précisons que la structure de la langue elle-même n'est pas sanctionnée par une note, dans le but précisément d'encourager la prise de risque. La plupart des énoncés comportent des erreurs (signalées en italique et expliquées entre parenthèses; nous ne signalons pas les fautes de frappe) comme dans l'exemple ci-dessous (à distance) :

2. Do you think that to be inconscient, it is ok for kill... (erreur de lexique, erreur de préposition)

Cependant, il n'y a pas de proportionnalité entre la portée ou la complexité de la contribution de l'étudiant et la forme linguistique. Nous verrons plus loin que malgré des erreurs de langue, le raisonnement des étudiants prend des formes variées. Rares sont les phrases réellement difficiles à comprendre, comme celle-ci (à distance) :

3. If everyone haven 't the same definition, it is a good debate, but it's to difficult, to say that "to do nothing " exist... Because when you are in your room, some of us say " I do nothing" and the others say " I rest" (utilisation du pluriel au lieu du singulier, mauvaise reprise du pronom)

Malgré les difficultés, le recours au français reste très marginal (ici dans la fenêtre de chat) :

4. $\quad<\mathrm{MR}>$ La question c'est bien savoir si ce qu'on pense est toujours vrai $<$ ld $>$ la question n'est pas de savoir so l'on peut penser a quelaue chose qui est toujours vrai dans l'absolu

$<$ AL $>$ No, M., it's to know IF you can think of at least ONE THING always true

$<\mathrm{ld}>$ like democracy is not a dictature for example

Aux obstacles linguistiques s'ajoutent des problèmes de gestion du temps en ce qui concerne la formation à distance, en dépit desquels les étudiants parviennent à se retrouver en ligne :

5. <ld > yES, BECAUSE i HAVE NOT A LOT OF TIME AND IT,S MY FIRST COME, SO... (erreur sur la négation et erreur de lexique) 
même si les rencontres se font parfois à partir du lieu de travail :

6. $\quad<\mathrm{DB}>$ all friends, i must leave the discussion i am at job ! (erreur de déterminant et erreur de lexique)

Malgré ces difficultés langagières (en présentiel et à distance) et des difficultés d'ordre matériel (à distance), les traces d'engagement dans la tâche sont présentes et prennent des formes diverses : traces d'autonomie (dans le cas de la formation à distance), modalité, complexité du propos.

\subsection{Autonomie des étudiants}

Les étudiants, même face à ces difficultés diverses, prennent en main leur apprentissage et on trouve dans le corpus des signes de cette autonomie. Ces traces ne concernent que les étudiants à distance. Les débats se déroulent avec ou sans la tutrice. Dans l'extrait ci-dessous, cinq étudiants se retrouvent de leur propre initiative. Ils se sont concertés pour être en même temps sur Internet car l'outil utilisé est synchrone. L'un d'entre eux a choisi trois thèmes de débat parmi une liste soumise par l'enseignante, et ils négocient ensemble en anglais quel sujet privilégier :

7. $<$ al $>$ I've selected 3 thunks so that we could chose more easily which one we want to debate.

$<$ mr $>$ The 3 for me

$<\mathrm{lt}>$ Thunk 3 it's ok for all????

$<\mathrm{ld}>$ ok for the 3

À un autre moment, un étudiant prend l'initiative de proposer un sujet en écho avec le sujet courant :

8. < $\quad$ fl> a interesting topic could be educability and liberty

Ici, les étudiants prennent l'initiative de commencer le débat en l'absence de la tutrice :

9. <NO > so Mrs Rémon is not here for the moment ???

$\langle\mathrm{cV}\rangle$ no

$<\mathrm{cV}>$ we can not start?

$<\mathrm{NO}>$ Perhaps she can't be here tonight ....

$<\mathrm{NO}>$ of course we can start

Nous mettons également les épisodes d'entraide sur le compte d'un engagement autonome dans la tâche :

10. $<\mathrm{ld}>\mathrm{i}$ don't how to connect bubbles

$<\mathrm{AL}>$ To link your box to the one you want to react, hold the shift button, right click on your box and move your mouse holding the click until you've reached your target (il manque une proposition)

Les exemples d'entraide et d'encouragement mutuel sont nombreux. S'il est possible de les mettre sur le compte de la distance et d'autres facteurs, et pas seulement sur l'engagement provoqué par les thématiques éthiques, on peut faire l'hypothèse que les aspects éthiques contribuent aussi à une dynamique motivationnelle d'ensemble : 
11. $<\mathrm{NO}>$ Pascale, don't stress, it's a useful habit to get into .... Tonight, you will be the best on the Drew !!! (ajout d'un déterminant)

L'autonomie se fait également sentir dans la régulation entre étudiants (régulation linguistique dans le deuxième cas) :

12. <AL> Or if I have another one, but the thing if to spend hours about choosing the topic....rather debate and communicate (utilisation erronée de l'infinitif)

13. <LT> WHO ARE IN BLUE??? (utilisation du pluriel au lieu du singulier) $<\mathrm{AL}>$ Yes who's in blue?

On peut rapprocher de cette autonomie la prise de distance qu'implique l'utilisation d'une métalangue dans le discours des étudiants. Cette distance est prise par rapport à leur propre niveau de langue (exemple $n^{\circ} 14$, en présentiel), par rapport à l'anglais en général (exemple $n^{\circ} 15$, à distance), par rapport au débat (exemple $n^{\circ} 16$, à distance), ou par rapport à l'outil (exemple $n^{\circ} 17$, à distance):

14. (How to explain in English a so deep question without the adequate words?) (place des constituants du groupe nominal)

15. <FP> I just realized now, but the english makes a difference between "white" and "blank" (déterminant inutile et oubli de majuscule)

16. $<\mathrm{FP}>$ It seems we are derivating to a new thunk: What does make something exist? (erreur sur la forme interrogative)

17. $<\mathrm{LT}>$ No more space for the debate... $<\mathrm{AL}>$ Yes it would seem there's a limit of space... If you want we can start another debate on group 15 ? (erreur de temps verbal)

\subsection{Modalité}

Que ce soit par le choix du lexique, des points d'exclamation, ou des marques d'humour, les apprenants prennent pleinement possession de leur discours autour de ces questions éthiques en y introduisant de la modalité. Nous entendons par « modalité » ce qui marque la présence du locuteur dans l'énoncé au sens où l'entend la linguistique de l'énonciation : "la modalité est au service du locuteur et lui permet de marquer sa présence de manière à marquer son attitude dans son énoncé » (Büyükgüzel $2011:$ 132).

\section{- Première personne : «I»/ «my »}

Par l'utilisation de la première personne, les étudiants (en présentiel) s'investissent dans leur réponse :

18. I can't do it because I will have some regrets in the future. I think that, whatever the pains that this persons caused to me, I can forgive. (confusion entre this/singulier et these/pluriel)

\section{- Lexique}

Dans certains extraits (exemple ${ }^{\circ} 19$, à distance), la motivation est tout simplement exprimée directement par le lexique (happy). L'objectif pédagogique de pratique de la 
langue est atteint : l'opposition entre cette modalité et la présence d'une erreur de langue est symptomatique d'un engagement dans la tâche en dépit des limitations linguistiques de l'étudiant.

19. <ld $>\mathrm{I}$ 'm very happy to be there !!! (confusion entre here et there)

Dans cet exemple (en présentiel), c'est aussi par le lexique (little joy) que l'énonciateur marque sa présence dans l'énoncé :

20. I don't fined my self sadic pearson but I am sure that I may fined a little joy in it. (orthographe)

\section{- $\quad$ Points d'exclamation}

La présence de points d'exclamation nous semble également montrer un investissement des apprenants dans la tâche qui leur est proposée. Les remarques des étudiants peuvent faire référence à des aspects pratiques comme ici dans la fenêtre de clavardage :

21. <al> Ho no! it's too early !!! Time flies too fast in these debates !

ou être directement liées à la réflexion éthique (à distance):

22. It's a real dilemma for me. In this time of crisis, we need time to think about it !!!

\section{- Humour}

Les marques d'humour sont nombreuses dans la fenêtre de clavardage, et il est intéressant de noter ici que si certains extraits sont des remarques concernant la logistique :

23. $<\mathrm{MJ}>$ Yes I'm here in the "choucroute" fish and chip ... (il manque la marque du pluriel)

d'autres portent bien sur le débat lui-même :

24. $\langle\mathrm{FP}>$ Who deleted some boxes???

$<\mathrm{ld}>$ MAYEBE OUR SUBSCONCIENT

Dans la fenêtre de débat, tous les extraits contenant des aspects humoristiques sont des moments du débat et non de la régulation :

25. So if a sleepwalker robs a fridge it is subconsciously bulimic (erreur sur le pronom personnel)

Tout comme dans les débats en ligne, les productions sur papier présentent des marques d'humour :

26. So I guess I would kindly ask the Mafioso to find someone else (and then report him to the supernatural police).

\subsection{Phraséologie}

Les éléments phraséologiques que l'on peut relever dans le corpus sont d'autres signes d'engagement de l'énonciateur dans son propos et typiquement d'une prise de risque langagière. Les étudiants tentent en effet d'employer des unités phraséologiques (sou- 
lignées) même si leur apprentissage n’est pas stabilisé (exemples $n^{\circ} 27$ et $n^{\circ} 28$, en présentiel ; exemple ${ }^{\circ} 29$, à distance).

27. But to my opinion just think of money and forget human being is a problem for me and personaly I don't want to sell my soul to devil just for money. (il manque -ing; il manque le déterminant)

28. My first choice that crosses my mind would be the director of Goldman Sachs bank who claimed to be the God of the world and setted up a whole strategy to ruin millions of people in purpose. It would be a pleasure! (erreur de déterminant ; erreur de prétérit ; erreur de préposition)

29. it is the principle of not seen not taken

Nous avons relevé jusqu'à présent dans le corpus des marques de la présence des étudiants dans leur discours que nous interprétons dans le sens d'un engagement dans la tâche. Ces traces sont complétées par des marques directes de motivation et des éléments que nous pouvons interpréter comme des signes d'autonomie, cela malgré les difficultés d'expression rencontrées par la plupart des apprenants, comme en témoignent de nombreuses erreurs et quelques recours au français. Nous souhaitons maintenant prendre des extraits du corpus qui montrent, malgré ces difficultés, la variété des opérations menées par les étudiants dans leur raisonnement.

\subsection{Complexité du propos}

En dépit d'erreurs linguistiques dans beaucoup d'extraits du corpus, le raisonnement offre une grande diversité d'opérations telles qu'établir une correspondance, structurer le propos, émettre une condition, établir une opposition, faire avancer le débat, donner un exemple, évoquer un principe.

\section{- $\quad$ Structurer son propos}

La structuration du propos peut se faire avec des connecteurs (en gras) (en présentiel) :

30. Moreover, noone would ever know. But I couldn't kill a person, even if I could earn many money. [...] Indeed, I'll choose probably somebody who made or makes bad things like Hitler. (erreur de déterminant)

ou par une structure physique (liste hiérarchisée, en présentiel) :

31. I would do it:

1) $[\ldots]$

2) $[\ldots]$

I wouldn't do it: $[\ldots]$

But people do inhuman things and what can we really do?

Cet aspect n'est pas présent dans le débat en ligne en raison de la structure hiérarchisée inhérente à la plateforme utilisée. Les flèches qui relient les boîtes font office de connecteurs.

\section{- Établir une correspondance ou une opposition entre deux notions}


Les participants au débat établissent des correspondances entre notions, par exemple entre amour et besoin (exemple $\mathrm{n}^{\circ} 32$, à distance), entre respirer et penser, entre dormir et agir, entre être malade et être irresponsable, entre penser et connecter des idées, entre quelque chose et rien, entre pensée et conscience, entre tuer et maintenir la paix (exemple ${ }^{\circ} 33$, en présentiel), etc. :

32. yes we can admit that in some areas loving and needing are the same (AE)

33. If you kill a guy who killed every day thousands of people it's a peace keeping act. (place de l'adverbe)

De même, les étudiants établissent des oppositions entre notions, par exemple entre objectif et subjectif, entre réel et imaginaire, entre besoin naturel et besoin vital, entre amour et besoin, entre physique et moral, entre violence et harcèlement (exemple $n^{\circ} 34$, à distance), entre homme et animal, entre parents et enfants, entre responsable et coupable, entre deux sphères (juridique / psychologique, professionnelle / scolaire), entre punition et internement, entre éducation et instruction, entre argent et récompense (exemple $n^{\circ} 35$, en présentiel), entre faire mal et torturer ou entre deux temporalités (exemple $n^{\circ} 36$, en présentiel), etc. :

34. violence and harassment is not quite the same! Comment : I think that violence is pretty front and harassment rather subtle NB (singulier au lieu du pluriel ; erreur de lexique)

35. Money don't be a reward. (erreur de construction verbale)

36. I think we can't do a real answer to this question, because we're not in this context right now. And this question need reflexion, but that's touching instinct, so our quick reaction. (erreur de lexique ; erreur de connecteur)

\section{- Émettre une condition : «if »}

Par l'utilisation de if (si), les étudiants distinguent deux situations, par exemple avec ou sans le consentement d'une personne (exemple n ${ }^{\circ} 37$, en présentiel), ou connaître ou non la personne et le degré de souffrance impliqués :

37. Even if it is with the consent of the person, I think that I'will not $d o$. (apostrophe inutile, manque le pronom)

\section{- $\quad$ Utiliser des références}

Un autre aspect des contributions des étudiants lors de ces débats sur des questions éthiques concerne la référence à des exemples (exemple $n^{\circ} 38$, à distance), parfois suivis sur plusieurs contributions, parfois inspirés de l'actualité (exemple ${ }^{\circ} 39$, à distance), à des exemples tirés de l'expérience personnelle (exemple $\mathrm{n}^{\circ} 40$, en présentiel et exemple $\mathrm{n}^{\circ} 41$, à distance) ou à des principes supérieurs (exemple $\mathrm{n}^{\circ} 42$, en présentiel) :

38. I'll give an example : If you're at work and are thinking (for your job), for any "outside" person you might be doing nothing.

39. do you remember all the people of France telecom who commited suicide? 
40. i think we can not, because I have earded by a member of my family a trange story [...] someone was in "coma', she didn't remember anything but when she waked-up, she said happy birthday to her son and it was the good day, so.... (erreur de participe passé ; erreur de prétérit)

41. know this in my job i $\mathrm{m}$ an estate agent ans my boss put a big pressure on me to make money and i m just fed up with this and this time i $m$ under pression just now (erreur de lexique)

42. I have principales and morals which doesn't allowed me to do to fell some one. (erreurs de lexique et singulier au lieu du pluriel)

\section{- $\quad$ Préciser, affirmer, conclure}

L'étude du corpus montre que les étudiants ne font pas qu'écrire des phrases déconnectées les unes des autres. Dans le cas du débat en ligne (car le travail en présentiel est individuel), ils tiennent compte de ce qui est dit par leurs pairs. Dans l'exemple cidessous (exemple $n^{\circ} 43$, à distance), l'étudiant fait avancer le débat en affirmant un point de vue :

43. Yes. And I believe we can have an obssesive idea without being ill of it (calqué du français)

\section{Conclusion}

Une étude de corpus de productions écrites d'étudiants en Master dans deux contextes différents nous a permis de relever des traces d'un engagement des apprenants dans la tâche, notamment à travers des marques énonciatives. Certains aspects de cet engagement sont synonymes de prise de risque langagière, telles les tentatives d'utilisation de phraséologie ou les marques d'humour. D'autres montrent une prise en main par les apprenants de leur apprentissage et une capacité à argumenter même avec des moyens linguistiques limités, comme en témoignent les nombreuses erreurs relevées dans les énoncés étudiés.

Le recours à des questions éthiquement vives n'est sans doute pas le seul facteur à l'origine des signes d'engagement dans la tâche relevés dans le corpus. Dans le cas du débat en ligne, par exemple, la motivation peut être due également à l'outil lui-même que les étudiants trouvent en général attrayant, même s'il présente quelques défauts. Cette motivation peut être générée d'une manière générale par l'activité de débat, indépendamment du thème. D'autre part, rappelons que ces étudiants sont en Master ; c'est donc un public sélectionné qui a choisi cette formation, même si leur charge professionnelle, familiale et universitaire est lourde durant cette année d'études. Signalons aussi que, dans le cas de la formation en ligne, certains étudiants sont géographiquement très distants. Dans un cas par exemple, un étudiant est au Japon, donc la connexion sur la plateforme de débat pour interagir avec ses pairs correspond à un réel besoin. C'est sans doute une combinaison de facteurs qui permet d'obtenir un investissement des étudiants, et ces questions provocatrices contribuent à une dynamique motivationnelle globale. Cependant, le caractère crucial des thématiques proposées aux étudiants, et des enjeux perçus à travers elles, invite les apprenants à 
se situer par rapport à des principes universels appelés plus que jamais à devenir un projet commun d'humanisation, d'une humanité qui aujourd'hui est plus souvent destructrice d'elle-même que constructrice de son avenir. [...] Le propre de l'humanité, c'est bien la capacité à partager l'humain, à défendre la communauté des hommes ici et maintenant. (Forestal $2007: 121-122$ )

Le caractère transversal des questionnements éthiques permet de faire de la classe de langue un des ancrages de ce projet d'humanisation mentionné par la citation de Forestal ci-dessus, et la communauté de confiance que l'on peut y créer fait écho à cette « communauté des hommes » dont la langue, fut-elle de spécialité, est bien le socle.

\section{Bibliographie}

Astolfi, J.-P. 2006. «Les questions vives en question ? Préface ». In Legardez, A. \& L. Simonneaux (dir.). L'école à l'épreuve de l'actualité, enseigner les questions vives. Paris : ESF.

Audigier, F. 1999. L'éducation à la citoyenneté. Lyon : Institut national de recherche pédagogique (INRP).

Büyükgüzel, S. 2011. «Modalité et subjectivité : Regard et positionnement du locuteur ». Synergies Turquie, $n^{\circ} 4: 139-151$. <http://ressources-cla.univfcomte.fr/gerflint/Turquie4/buyukguzel.pdf> (consulté le 26 octobre 2012).

Forestal, C. 2007. «La dynamique conflictuelle de l'éthique : pour une compétence éthique en DLC ». Étude Linguistique Appliquée, $n^{\circ} 145: 111-123 .<$ http://www.aplvlanguesmodernes.org/IMG/pdf/Forestal_competence_ethique_DLC.pdf $>$ (consulté le 26 octobre 2012).

Forestal, C. 2009. « La démarche transculturelle en Didactique des Langues-Cultures : une démarche discutable... et/ou qui mérite d'être discutée ». Synergies Pays Riverains de la Baltique, $n^{\circ} 6$ : 59-75. <http://ressources-cla.univfcomte.fr/gerflint/Baltique6/forestal.pdf> (consulté le 26 octobre 2012).

Gilbert, I. 2007. The Little Book Of Thunks. Carmarthen: Crown House Publishing.

Legardez, A. \& L. Simonneaux. (dir.) 2006. L'école à l'épreuve de l'actualité. Enseigner les questions vives. Issy-les-Moulineaux : ESF.

Mathieu, A. 2010. «Pratique d'une pédagogie de l'étonnement en mathématiques en classe de seconde ». Expressions, $n^{\circ} 35: 93-118$.

Puren, C. 1994. «Éthique et didactique scolaire des langues ». Les Langues modernes, $n^{\circ} 3: 55-62$.

Rémon, J. 2010. «Online mind maps for language learning: Assisted Risk-Taking ». In Gimeno Sanz, A. (ed.). New trends in Computer-Assisted Language Learning : working together, Proceedings of the annual conference of the European Association for Computer-Assisted Language Learning (EUROCALL 2009). MacMillan. 
Rémon, J. 2012. "Supports filmiques transversaux en pratique de la langue : document authentique ou authentiquement intéressant ? ", Recherche et pratiques pédagogiques en langues de spécialité, vol. XXXI, $n^{\circ} 2: 90-102$.

Scrivener J. 2011. Learning teaching, Oxford: MacMillan.

Simonneaux, J. \& A. Legardez. 2010. «The Epistemological and Didactical Challenges Involved in Teaching Socially Acute Questions ». Journal of Social Science Education, vol. $9, n^{\circ} 4$ : 24-35 <http://www.jsse.org/2010/2010-4/pdf/Simonneaux-et-alJSSE-4-2010.pdf> (consulté le 26 octobre 2012).

\section{Annexe : traduction des extraits du corpus ${ }^{3}$}

1. $<\mathrm{jm}>\mathrm{j}$ 'admets que ce sujet ne m'inspire pas ! Désolée $<\mathrm{cv}>$ pareil pour moi... mais c'est juste pour l'exercice... $<\mathrm{jm}>$ oui bien-sûr!

2. Est-ce que vous croyez qu'être inconscient, c'est ok de tuer...

3. Si tout le monde n'ont pas la même définition, c'est un bon débat, mais c'est trop difficile de dire que «ne rien faire » existe... Parce que quand vous êtes dans votre chambre, certains d'entre nous disent « je ne fais rien » et les autres disent « je me repose»

4. $<$ AL $>$ Non, M., c'est de savoir SI tu peux penser au moins à une chose toujours vraie $<\mathrm{ld}>$ ccomme la démocratie n'est pas une dictature par exemple

\section{5. < ld > Oui, PARCE QUE JE N'AI PAS BEAUCOUP DE TEMPS ET C'EST MA} PREMIERE VENUE, DONC...

6. $\angle \mathrm{DB}>$ tous les amis, je dois quitter la discussion je suis au travail !

7. $<\mathrm{al}>\mathrm{j}$ 'ai sélectionné 3 thunks pour que nous puissions choisir plus facilement celui dont nous voulons débattre.

$<\mathrm{mr}>$ Le 3 pour moi

$<\mathrm{lt}>$ Thunk 3 est ok pour tous ????

$<\mathrm{ld}>$ ok pour le 3

8. <fl > un sujet intéressant pourrait être éducabilité et liberté.

9. $<\mathrm{NO}>$ alors Mme Auteur n'est pas là pour le moment ???

$<\mathrm{cV}>$ non

$<\mathrm{cV}>$ nous ne pouvons pas commencer?

$<\mathrm{NO}>$ Peut-être qu'elle ne peut pas être là ce soir....

$<\mathrm{NO}>$ bien-sûr nous pouvons commencer

10. $<\mathrm{ld}>$ je ne sais pas comment connecter les bulles

$<\mathrm{AL}>$ Pour relier ta boîte à celle que tu veux réagir, garde la touche shift appuyée, fais un clique droit sur ta boîte et bouge la souris en maintenant le clique jusqu'à ce que tu aies atteint ta cible

\footnotetext{
${ }^{3}$ Nous avons, quand c'était possible, conservé en français des erreurs équivalentes à l'original.
} 
11. <NO > Pascale, ne stresse pas, c'est une habitude utile à prendre.... Ce soir, tu seras la meilleure sur le Drew !!!

12. $<$ AL $>$ Où si j'en ai un autre, mais le problème si passer des heures à choisir le sujet... plutôt que débattre et communiquer

13. <LT> QUI SONT EN BLEU ???

$<\mathrm{AL}>$ Oui qui est en bleu?

14. (Comment expliquer en anglais une si profonde question sans les mots adéquats ?)

15. $<\mathrm{FP}>\mathrm{Je}$ viens de m'en rendre compte, mais l'anglais distingue « white » et «blank »

16. $<\mathrm{FP}>$ On dirait que nous dérivons vers un nouveau thunk : qu'est-ce qui fait que quelque chose existe?

17. $<\mathrm{LT}>$ Plus de place pour le débat...

$<\mathrm{AL}>$ Oui cela semblerait qu'il y a une limite de place... Si vous voulez nous pouvons démarrer un autre débat sur le groupe 15 ?

18. Je ne peux pas le faire parce que $\mathrm{j}$ 'aurai des regrets dans le futur. Je crois que, quelles que soient les souffrances que cette personne m'a infligées, je peux pardonner.

19. $<\mathrm{ld}>$ je suis très contente d'être là !!!

20. Je ne me considère pas une personne sadique mais je suis sure que je pourrais trouver un peu de joie à faire là-dedans.

21. <al> Ho non ! il est trop tôt !!! Le temps passe trop vite dans ces débats !

22. C'est un vrai dilemme pour moi. En ce temps de crise, on a besoin de temps pour y penser !!!

23. $<\mathrm{MJ}>$ Oui je suis ici dans la "choucroute" fish and chip ...

24. <FP> Qui a effacé des boîtes???

$<$ ld $>$ PEUT-ÊTRE NOTRE SUBSCONCIENT

25. Donc si un somnambule dévalise un frigo il est inconsciemment boulimique

26. Donc j'imagine que je demanderais gentiment au Mafioso de trouver quelqu'un d'autre (et ensuite le dénoncer à la police surnaturelle).

27. Mais à mon avis juste penser à l'argent et oublier l'être humain est un problème pour moi et personnellement je ne veux pas vendre mon âme à diable juste pour de l'argent.

28. Mon premier choix qui me traverse l'esprit serait le directeur de la banque Goldman Sachs qio prétendait être le Dieu du monde et a mis au point une stratégie complète pour ruiner des millions de personnes exprès. Ca serait un plaisir !

29. C'est le principe de pas vu pas pris

30. De plus, personne ne le saurait jamais. Mais je ne serais pas capable de tuer quelqu'un, même si je pouvais gagner de nombreux argent. [...] En effet, je choisirai probablement quelqu'un qui fait ou a fait de mauvaises choses comme Hitler.

31. Je le ferais :

1) [...] 
2)[...]

Je ne le ferais pas : $[\ldots]$

Mais les gens font des choses inhumaines et que peut-on vraiment faire?

32. Oui nous pouvons admettre que dans certains domaines l'amour et le besoin sont identiques (AE)

33. Si vous tuez quelqu'un qui a tué tous les jours des milliers de personnes c'est un acte de maintien de la paix.

34. La violence et le harcèlement ne sont pas tout à fait la même chose ! Commentaire : Je crois que la violence est plutôt frontale et le harcèlement plutôt subtil NB

35. L'argent ne pas être une récompense.

36. Je crois que nous ne pouvons faire une vraie réponse à cette question, parce que nous ne sommes pas dans ce contexte en ce moment précis. Et cette question demande réflexion, mais ça concerne l'instinct, donc notre réaction rapide.

37. Même si c'est avec le consentement de la personne, je crois que je ne ferais pas.

38. Je donne un exemple : Si on est au travail et qu'on réfléchit (au travail), pour une personne « extérieure » on pourrait être en train de ne rien faire.

39. Vous vous rappelez toutes les personnes à France telecom qui se sont suicidées ?

40. Je crois qu'on ne peut pas, parce que j'ai entendu par un membre de ma famille une étrange histoire [...] quelqu' un était dans le coma, elle ne se souvenait plus de rien mais quand elle s'est réveillée, elle a dit joyeux anniversaire à son fils et c'était le bon jour, alors....

41. Je connais ça dans mon travail je suis agent immobilier et mon patron me mets une grosse pression pour faire de l'argent et j'en ai marre et cette fois je suis sous pression en ce moment même

42. J'ai des principes et des morales qui ne me permirent pas de faire mal à quelqu'un.

43. Oui. Et je crois qu'on peut avoir une idée obsessive sans en être malade

Après des études en linguistique et informatique, Joséphine Rémon a été attachée linguistique à l'ambassade de France à Ottawa (Canada). Elle a consacré son doctorat à créer et implémenter des scénarios ludiques pour faire se rencontrer linguistique, apprentissage des langues et Internet. Elle enseigne l'anglais et les TIC à l'université Lyon 2, aux étudiants anglicistes et en Master Sciences de l'éducation et Master Humanitaire et poursuit ses recherches au sein du laboratoire ICAR (UMR5191).

<josephine.remon@univ-lyon2.fr> 